\title{
Meiotic instability associated with the CAGR1 trinucleotide repeat at $13 \mathrm{q} 13$
}

\author{
Nicholas T Potter
}

\begin{abstract}
CAGR1 is a recently characterised polymorphic trinucleotide repeat localised to 13q13, which has been suggested as a possible candidate gene for neurological disorders that manifest genetic anticipation. To provide evidence in support of this hypothesis, a large number of chromosomes $(n=928)$ from patients with a wide variety of neurological diseases were screened for evidence of repeat expansion and meiotic instability. One person with a CAGR1 repeat number of 50 was identified (normal range 9-29). Subsequent molecular analyses of CAGR1 repeat number in additional family members showed meiotic instability of a (CAG) ${ }_{45}$ allele through three generations. While CAGR1 repeat number did not correlate with a readily discernible phenotype in this family, the finding of meiotic stability and mendelian inheritance of normal CAG alleles and meiotic instability of larger repeats fulfil several criteria thought essential for pathologically relevant mutations of this type. Thus, these data strengthen the hypothesis for a role of CAGR1 in the development of an as yet molecularly uncharacterised human neurological disease.

(F Med Genet 1997;34:411-413)
\end{abstract}

Keywords: CAGR1; trinucleotide repeat; meiotic instability

The expansion of reiterated CAG sequences is associated with phenotypic expression in at least six inherited neurological disorders ${ }^{1}$ and mutations of this type are suspected candidates for several other neuropsychiatric and neurodegenerative disorders which manifest genetic anticipation..$^{2-4}$ Efforts to identify and characterise candidate genes containing trinucleotide repeats have generally relied upon the screening of human brain cDNA libraries and human expressed sequence tags (ESTs) and the use of repeat expansion detection (RED) or FISH for the identification of abnormally long or highly polymorphic sequences, ${ }^{5-10}$ and the causative mutation in at least one neurodegenerative disease, dentatorubral-pallidoluysian atrophy, was subsequently identified using one of these approaches. ${ }^{611} 12$ Recently Margolis et $a l,{ }^{13}$ screening a human retinal cDNA library, identified a highly polymorphic CAG repeat (called CAGR1) contained within the 5' untranslated region of a gene that shares sequence homology with the Caenorhabditis elegans cell fate determining protein mab- 21 . The highly polymorphic nature of this repeat (normal repeat range from 6-31 copies), its high observed heterozygote frequency, its predominant expression in brain (cerebellum), and their subsequent identification of a single allele with 46 repeats in a person with an idiopathic movement disorder and an affective disorder provide compelling arguments for consideration of this locus as a candidate gene for molecularly uncharacterised inherited neurodegenerative, neuropsychiatric, or neurodevelopmental disorders. Whereas previous studies have elucidated the polymorphic nature of this repeat, ${ }^{13}{ }^{14}$ there have not been any reports on the identification of meiotically unstable CAGR1 alleles, an essential feature for any trinucleotide repeat locus presumed to be involved in the aetiology of disorders of this type.

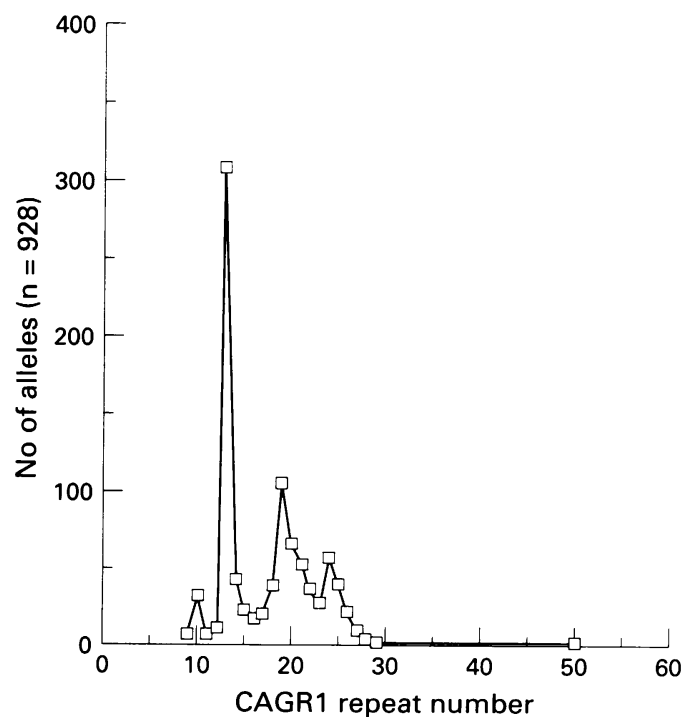

Figure 1 Distribution of CAGR1 repeat length from the 928 alleles. (CAGR1) ${ }_{13}$ represented the most common allele ( $33 \%$ of all alleles tested) followed by (CAGR1) $(11 \%$ of alleles). Excluding the one patient with the (CAGR1) there were no differences in allele distribution between the nine different patient groups. 

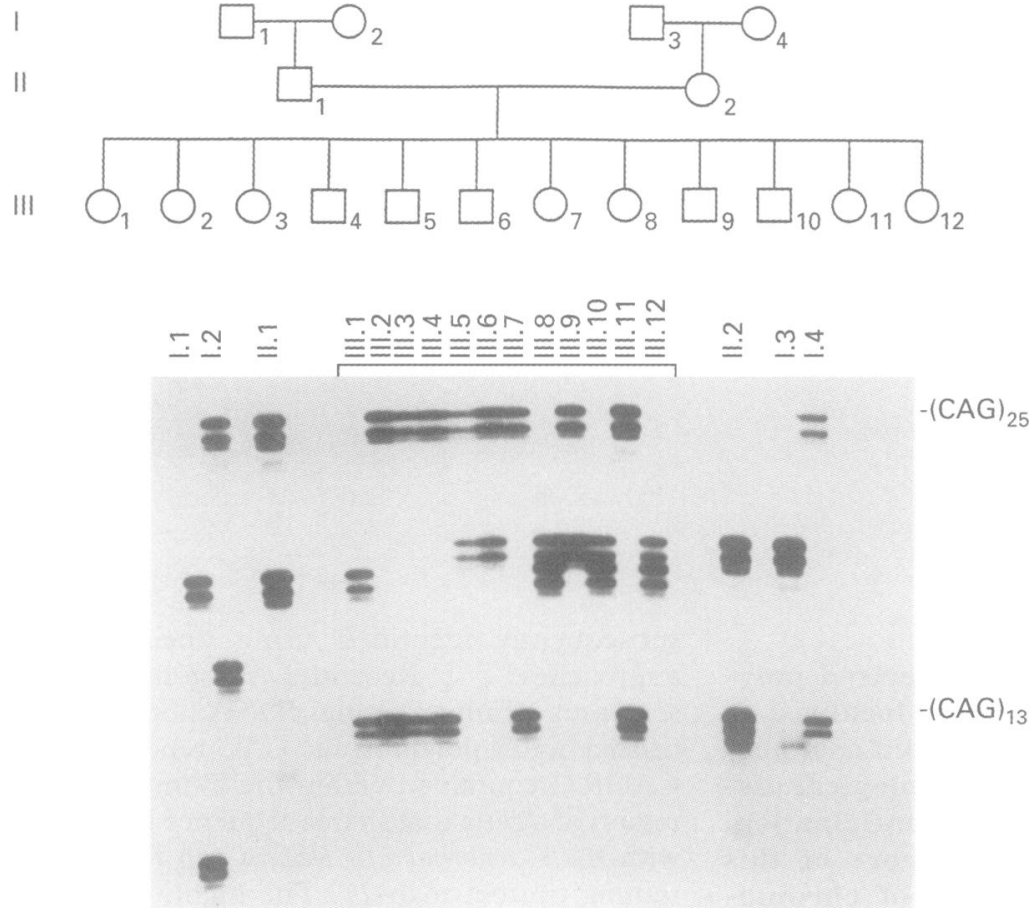

Figure 2 Six percent sequencing gel analysis showing meiotic stability and mendelian inheritance of CAGR1 repeats in the normal range through 14 meioses in the CEPH/Amish pedigree 884. PCR primers for CAGR1 and conditions for analysis were essentially as described in Margolis et al ${ }^{13}$ with the exception that ${ }^{32} P-d C T P$ was incorporated into the reaction and the annealing temperature for CAGFM-5'/CAGFM-3' (10 pmol of each $/ 25 \mu \mathrm{l}$ reaction) was lowered to $50^{\circ} \mathrm{C}$. Similar analysis of the segregation of a stable $(C A G)_{20}$ repeat through five meioses was also performed on DNA samples obtained from family DE0005 (data not shown). Allele sizes were as follows: I.1 (10/19);I.2 (16/25);II.1 (19/25); III. (13/19); III. 2 (13/25); III. 3 (13/25);III. 4 (13/25); III. 5 (20/25);III. 6 (20/25); III. 7 (13/25);III. 8 (19/20); III.9 (20/25);III. 10 (19/20); III.11 (13/25);III. 12 (19/20);II. 2 $(13 / 20) ; 1.3$ (20/20);I.4 (13/25). DNA samples from the CEPH/Amish pedigree 884 and family DE0005 were obtained from the NIGMS Human Genetic Mutant Cell Repository, Coriell Institute for Medical Research, Camden, NF 08103.
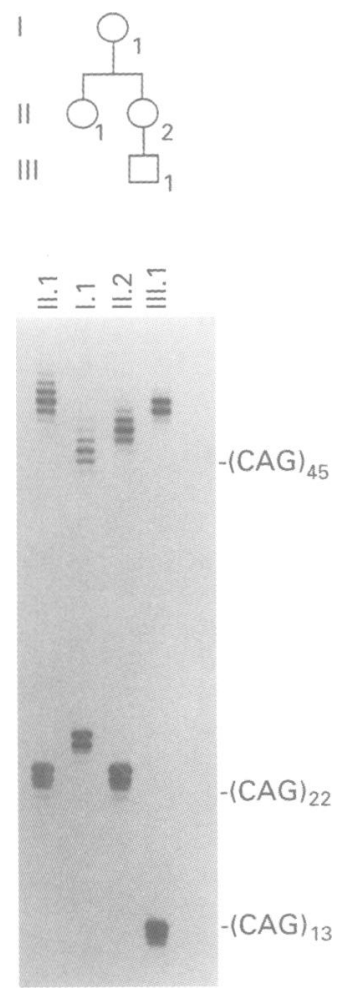

Figure 3 Meiotic instability of a CAGR1 repeat greater than 45 through three meioses. DNA was isolated from peripheral blood lymphocytes. Allele sizes: I. 1 (24/45);II. 1 (22/51);II.2 (22/47); III.1 (13/50). Assay conditions were as described in fig 2 .
In an effort to identify such alleles, a total of 928 chromosomes were studied for the determination of CAGR1 repeat number. This included people with inherited neurodegenerative disorders of unknown aetiology who tested negative for $C A G$ expansions at the $\mathrm{HD}$, DRPLA, SCA-1, SCA-2, and SCA-3 loci $(n=62)$, patients with Parkinson's disease $(n=14)$, progressive supranuclear palsy $(n=6)$, multiple system atrophy $(n=6)$, nigrostriatal degeneration $(n=6)$, multiple sclerosis $(n=10)$, Alzheimer's disease $(n=17)$, molecularly negative fragile XA patients with developmental delay/mental retardation $(n=295)$, and normal controls $(n=48)$. The distribution of normal CAG repeat size in this cohort was 9-29 (fig 1), with a modal repeat length of 13 and heterozygosity of $89 \%$. One person from the fragile XA negative group was found to have an allele with 50 CAGR1 repeats (fig 3, III.1). As this allele was almost twice the size of the largest normal allele found in this cohort, additional DNA samples were obtained from other family members to examine for evidence of meiotic instability. In contrast to the stable inheritance of CAG repeat lengths in the normal range (fig 2 ), this family clearly showed meiotic instability for CAGR1 alleles of 45 or larger with a $\triangle \mathrm{CAG}$ of at least five repeats observed through three generations (fig 3).

Although the index case in this family had a phenotype of idiopathic mental retardation with an emergent attention deficit/ hyperactivity disorder, there are several reasons why it may be premature to presume his phenotype is related to the presence of the (CAGR1 $_{50}$ allele. First, and most significant, is the lack of any readily discernible phenotype in his grandmother, mother, or aunt despite the presence of similarly sized CAGR1 alleles. Second, the localisation of the repeat in an untranslated region of the gene, the small increase in CAG length over three generations in a manner consistent with the "slippage mediated model" for repeat sequence instability, ${ }^{15}$ and the finding of meiotically unstable alleles in the 40-50 range collectively suggest that the CAGR1 expansion has molecular characteristics reminiscent of the premutations found in the type II repeat expansion mutation diseases fragile $\mathrm{XA}$ and $\mathrm{E}$, myotonic dystrophy, and Jacobsen syndrome. ${ }^{16}$ Whether CAGR1 alleles are prone to further expansion into a pathological size range is currently unknown, although our finding of CAGR1 locus instability and the recent identification of a large $(3.0 \mathrm{~kb})$ expansion in a novel CAG repeat found in the non-coding region of HC 18q21-22 segregating in a family with schizophrenia ${ }^{17}$ suggests that larger expansions are possible.

In summary, our data provide evidence of meiotic stability for normal sized CAGR1 alleles and meiotic instability for larger alleles. Collectively these data strengthen the possibility that CAGR1 expansion may be involved in the development of an as yet molecularly uncharacterised human neurological disease. 
I would like to thank Richard Greene, MD and Virginia Frye, EdD for their assistance with the evaluation of the index case and Enid Bowlin for her technical assistance with the fragile XA studies. I would also like to thank the other family members for their participation in this study. Postmortem tissue samples were obtained from the Harvard Brain Tissue Resource Center, Belmont, MA 02178 which is supported by NIH award RO1-MH31862 and the National Neurological Research Specimen Bank, VAMC, Los Angeles, CA 90073, which is sponsored by NINDS/NIMH, National Multiple Sclerosis Society, Hereditary Disease Foundation, Comprehensive Epilepsy Program, Tourette Syndrome Association, Dystonia Medical Research Foundation, and Veterans Health Services and Research Administration, Department of Veterans Affairs. This study was supported in part by grants from the Physicians' Medical and Education Research Foundation (PMERF), Knoxville, TN and the State of Tennessee.

1 Zoghbi HY. The expanding world of ataxins. Nat Genet 1996;14:237-8.

2 Ross CA, McInnis MG, Margolis RL, Li SH. Genes with triplet repeats: candidate mediators of neuropsychiatric disorders. Trends Neurosci 1993;16:254-60.

3 O'Donovan MC, Guy C, Craddock N, et al. Expanded CAG repeats in schizophrenia and bipolar disorder. Nat Genet 1995;10:380-1.

4 Junck L, Fink JK. Machado-Joseph disease and SCA3: the genotype meets the phenotypes. Neurology 1996;46:4-8.

5 Riggins GJ, Lokey LK, Chastain JL, et al. Human genes containing polymorphic trinucleotide repeats. Nat Genet containing poly

$6 \mathrm{Li} \mathrm{SH,} \mathrm{McInnis} \mathrm{MG,} \mathrm{Margolis} \mathrm{RL,} \mathrm{Antonarakis} \mathrm{SE,} \mathrm{Ross}$ CA. Novel triplet repeat containing genes in human brain: cloning, expression, and length polymorphisms. Genomics 1993;16:572-9.

7 Jiang JX, Deprez RHL, Zwarthoff EC, Riegman PHJ. Characterization of four novel CAG repeat-containing clones. Genomics 1995;30:91-3.
8 Adams MD, Kervalage AR, Fleishmann RD, et al. Initial assessment of human gene diversity and expression patterns based upon 83 million nucleotides of cDNA sequence. Nature 1995;377:3-47.

9 Schalling M, Hudson TJ, Buetow KH, Houseman DE. Direct detection of novel expanded trinucleotide repeats in the human genome. Nat Genet 1993;4:135-9.

10 Haaf T, Sirugo G, Kidd KK, Ward DC. Chromosomal localization of long trinucleotide repeats in the human genome by fluorescence in situ hybridization. Nat Genet 1996;12:183-5.

11 Koide R, Ikeuchi T, Onodera O, et al. Unstable expansion of CAG repeat in hereditary dentatorubral-pallidoluysian atrophy (DRPLA). Nat Genet 1994;6:9-13.

12 Nagafuchi S, Yanagisawa $\mathrm{H}$, Sato $\mathrm{K}$, et al. Dentatorubral and pallidoluysian atrophy expansion of an unstable CAG trinucleotide on chromosome 12p. Nat Genet 1994;6:1418.

13 Margolis RL, Stine OC, McInnis MG, et al. cDNA cloning of a human homologue of the Caenorhabditis elegans cell fate-determining gene mab-21: expression, chromosomal location and analysis of a highly polymorphic (CAG)n trinucleotide repeat. Hum Mol Genet 1996;5:607-16.

14 Neri C, Albanese V, Lebre AS, et al. Survey of CAG/CTG repeats in human cDNAs representing new genes: candidates for inherited neurological disorders. Hum Mol Genet 1996;5:1001-9.

15 Richards RI, Sutherland GA. Simple repeat DNA is not replicated simply. Nat Genet 1994;6:114-16.

16 Warren ST. The expanding world of trinucleotide repeats. Science 1996;271:1374-5.

17 Breschel TS, Sirugo G, Pleasant N, et al. A novel, heritable, expanding CAG repeat in a non-coding region of $\mathrm{HC}$ 18q21-22 in CEPH and bipolar pedigrees. Am $\mathcal{F}$ Hum Genet 1996;59:A214. 